\title{
Tactical Surface Metering Procedures and Information Needs for Charlotte Douglas International Airport
}

\author{
Savita Verma ${ }^{1}$, William J. Coupe ${ }^{2}$, Hanbong Lee ${ }^{1}$, Isaac Robeson ${ }^{3}$, \\ Yoon Jung ${ }^{1}$, Shivanjli Sharma ${ }^{1}$, Victoria L. Dulchinos ${ }^{4}$, Lindsay Stevens ${ }^{1}$ \\ ${ }^{1}$ NASA Ames Research Center, Moffett Field, CA 94035, ${ }^{2}$ USRA, NASA Ames \\ Research Center, Moffett Field, CA 94035, ${ }^{3}$ Mosaic ATM, Leesburg, VA 20176, ${ }^{4}$ San Jose \\ State University, NASA Ames Research Center, Moffett Field, CA 94035 \\ \{Savita.A.Verma, William.J.Coupe, Hanbong.Lee, Isaac.Robeson, Yoon.Jung, \\ Shivanjli.Sharma, Victoria.L.Dulchinos, Lindsay.Stevens\} @ nasa.gov
}

\begin{abstract}
NASA has been working with the FAA and aviation industry partners to develop and demonstrate new concepts and technologies that integrate arrival, departure, and surface traffic management capabilities. In the fall of 2017, NASA began deployment of their technologies in a phased manner to assist with the integrated surface and airspace operations at Charlotte Douglas International Airport (Charlotte, NC). Initial technologies included a tactical surface metering tool and data exchange elements between the airline-controlled ramp and Federal Aviation Administration controlled ATC Tower. In this paper, we focus on the procedures associated with the tactical surface metering tool used in the ramp control tower. This tool was first calibrated in Human-In-the-Loop simulations and was further developed when it was used in the operational world. This paper describes the collaborative procedures that the users exercised in their respective operational worlds to enable surface metering and how several metrics were used to improve the metering algorithm.
\end{abstract}

Keywords: Human Factors · Surface Metering $\cdot$ Collaborative decision making

\section{Introduction}

Managing departure operations in busy airport and airspace environments, with limited data sharing and system integration, can significantly limit efficiency and predictability. Stakeholders, including air carriers and air navigation service providers, have their own objectives in managing traffic, and often these objectives compete with one another. Also, decisions in managing traffic are often made in a reactive manner with short planning horizons due to operational uncertainties and a lack of common situation awareness between Flight Operators and service providers. For example, during periods when demand exceeds capacity at the airport, service providers manage traffic using the First Come, First Serve (FCFS) paradigm, where they serve the flights that first call in as ready for pushback. With many airlines having similar ticketed departure times, this leads to surface congestion. There is a need for a tool that provides departure metering to prevent surface congestion. Other tools that perform departure metering include 
those deployed at sites such as John F. Kennedy (JFK) airport (NY) and are focused on providing Target Movement Area entry Times (TMATs) to the airlines several hours in advance, making the tool primarily strategic in nature [1]. The tool deployed at JFK provides departure metering capability with a longer planning horizon, i.e., several hours into the future, and it also allows the airline users to update flight ready times and request flight swaps as they know the situation better. A departure metering tool that could meter the traffic while considering arrivals and runway crossings, in a tactical manner, could potentially alleviate the congestion problem. Research on one such tool, the Spot and Runway Departure Advisor (SARDA) [2, 3] has been conducted at NASA Ames Research Center. This tool was further investigated and deployed at Charlotte Douglas International Airport (CLT), NC in the fall of 2017 under the Airspace Technology Demonstration 2 (ATD-2) project [4] described in the next section.

The National Aeronautics and Space Administration (NASA) has been collaborating with the Federal Aviation Administration (FAA) and aviation industry partners to develop and demonstrate new concepts and technologies to solve some of these complex problems in the Integrated Arrival, Departure, and Surface (IADS) traffic management capabilities under the ATD-2 project. The primary goal of the ATD-2 project is to improve the predictability and the operational efficiency of the air traffic system in metroplex environments while maintaining or improving throughput by enhancing and integrating arrival, departure and surface prediction, scheduling, and management systems. The IADS capabilities defined in the ATD-2 project are built upon previous NASA research, including the Spot and Runway Departure Advisor (SARDA) [2, 3], the Precision Departure Release Capability (PDRC) [5], and the Terminal Sequencing and Spacing (TSAS) capability [6]. In the first phase of deployment in CLT, a tactical surface scheduler was deployed at CLT in the ramp control facility. The initial integrated system of tactical surface scheduling deployed incorporated Surface Collaborative Decision Making (S-CDM) [7] principles and tactical departure scheduling to an enroute meter point that will help insert departures into the overhead departure stream. This paper will focus on the procedures and information requirements for the Tactical Surface Scheduler, also referred to as the Metering Tool.

The Metering Tool described in this paper is tactical in nature and works over a relatively short time horizon. It is meant to provide the airline ramp controller with aircraft pushback advisories that reduce surface congestion and to respond to surface and airspace constraints that become known with greater certainty in the next 10 minutes. The ramp controllers, in general aim to push flights as they call in, which is the biggest cause of surface congestion. They do so because it keeps their workload manageable and also vacates the gates for arrivals. Thus, the tactical surface metering tool first estimates the capacity of current and near-future runway resources from flight schedule and surveillance data. With demand forecasts and predicted taxi trajectories, this tool computes an efficient runway schedule of aircraft within the planning horizon based on their flight readiness as indicated by Earliest Off-Block Times (EOBTs), according to a ration by schedule (RBS) rule. The tool then generates runway schedules and recommended hold time advisories to meet the runway schedule. These advisories are shown on the user interfaces for the ramp controller and the ramp traffic manager, called Ramp Traffic Console (RTC) and Ramp Manager Traffic Console (RMTC), respectively. RTC and RMTC were developed as part of the SARDA project [3]. 
A Human-in-the-loop (HITL) study investigated procedures for the Metering Tool [8] and found that ramp managers may have RTMC running all the time, and they can enable or disable metering anytime, depending on their strategy for demand/capacity balancing. When ramp managers decide to enable the time-based metering, they could choose the level of gate holding from three options - 'Nominal hold', 'Less hold', or 'More hold.' The 'Less hold' option allows more flights to be on the airport surface (movement area), whereas the 'More hold' option allows the flights to be held at their gates longer, thus resulting in less taxiing delay or less excess queue time on the surface or airport movement area. The 'Nominal hold' option seeks to utilize the existing runway capacity with the available demand and find acceptable levels of excess queue time taken on the surface. These gate hold levels are associated with a metering value that defines the target excess queue time that will be taken in the Airport Movement Area (AMA) as compared to unimpeded transit time from gate to runway. A study [8] found that users preferred the inputs for level of gate hold to be labeled as "Excess Queue Time in AMA" and they chose $12 \mathrm{~min}$ as the target for excess queue time with $14 \mathrm{~min}$ to mark "less hold" and 10 min to represent "more hold" as shown in Fig. 3.

In this paper, we describe the procedures that were adopted for tactical surface metering and the way these evolved. In section 2 , we will briefly overview the airport surface and airspace operations at CLT. In section 3, we will describe the tools and interfaces deployed at CLT. In section 4 we will discuss data analysis of procedures and early anecdotal feedback, which led to changes to procedures and the algorithm that were adopted by the users during the field demonstration. Lastly, section V will provide the closing remarks.

\section{CLT Operations Overview}

According to the recent airport activity report, CLT accommodates about 1,400 operations per day and is the seventh busiest airport in aircraft movements worldwide in 2016 [9]. Because CLT is one of the main hub airports for American Airlines (AAL), AAL and its regional air carriers operate nearly $93 \%$ of the flights into and out of the airport. The remaining operations are comprised of other regional carriers, mainline flights operated by Southwest, Delta, United and Jet Blue, military flights, business and general aviation, and air cargo. As the dominant carrier, AAL manages all ramp operations at the airport, whereas air traffic on the AMA is controlled by the ATC Tower.

As shown in Fig. 1, CLT has three north/south parallel runways (18L/36R, 18C/36C, and $18 \mathrm{R} / 36 \mathrm{~L}$ ) that can support simultaneous independent instrument approaches, and a fourth diagonal runway $(5 / 23)$ that intersects Runway $18 \mathrm{~L} / 36 \mathrm{R}$. The airport operates in either a "North" or "South" flow configuration. The diagonal runway, Runway 23, is used in a South flow configuration for arrivals. Runway 5 (the opposing end) is not used for arrivals or departures during normal daylight/evening operations, but it is used as a taxiway in a North flow operation. However, during North flow operations, Runway 5 is used for both arrivals and departures when North flow nighttime noise abatement procedures are in effect.

Traffic at CLT is characterized by definite peaks and valleys. There are clear distinctions between departure and arrival banks throughout the day. Banks are peak periods of departure or arrivals due to the hub-and-spoke operations. Each departure and 
arrival bank takes approximately an hour with a slight overlap existing between banks. Ramp Control strives to clear the departures from the gates before an arrival bank builds up, so that ramp congestion and gate conflicts can be minimized. The ramp area is divided into four sectors (e.g., West, South, East, and North sectors). The corresponding ramp controller controls the traffic in each sector. The ramp operations at CLT are constrained due to physical limitations of the ramp, such as limited ramp space with alleys between concourses, single-direction taxiways, and limited holding areas (hardstands).

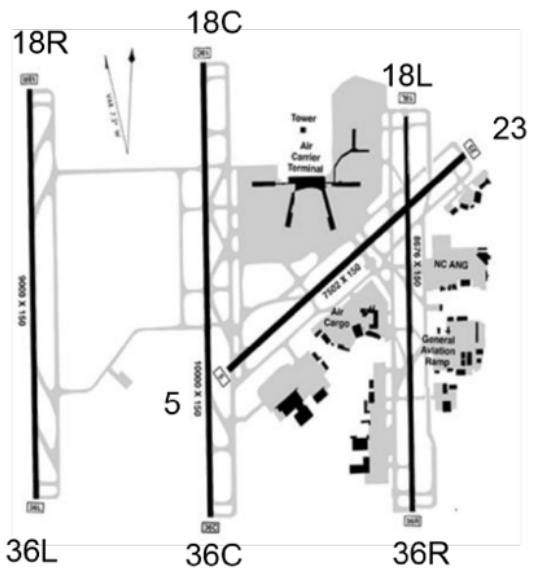

Fig. 1. CLT airport plan view

\section{$3 \quad$ Metering Tool User Interfaces and Procedures}

This section describes the Metering Tool's interface and procedures it was deployed at CLT in the fall of 2017. The metering tool was used by ramp traffic controllers and managers and to depict the advisories associated with departure metering.

\subsection{Ramp Traffic Console (RTC)}

The Ramp Traffic Console (RTC) and Ramp Manager Traffic Console (RMTC) are decision support tools developed for the ramp controllers and ramp managers, respectively. These tools provide a map display that depicts the ramp area with flight strips positioned at each gate for departures. These flight strips provide information such as call sign, aircraft type, and departure fix. The ramp controllers can interact with the tool to provide flight intent information, such as pushback, holding a flight, changing the assigned spot (point where control is handed over to FAA controlled from the ramp/airline), changing its gate, and marking the flight if it is sent to the hardstand. Double clicking on the flight strip allows the user to open the Flight Menu where the user can change assignment of a flight's spot, gate, runway, and mark it as temporarily out of service or mark it as being sent to the hardstand. Gate pushback intent information can also be provided by the ramp controller by right clicking the flight and selecting pushback in the drop-down menu, which is marked "pushback-cleared" with an engine 
symbol. Similarly, the right click drop down menu provides the user with an option to hold the flight by putting a red border around the flight strip (see Fig. 2). The color of the flight strips and icons show the flight direction they are going to, the blue strips have destinations in the east direction whereas the brown are flying in the west direction. Arrivals are depicted as green color aircraft icons.

Flights that are moving and tracked are shown as solid aircraft icons and those that are moving but not detected by surveillance are shown as hollow aircraft icons (see Fig. 2). Tactical Surface Scheduler/ Metering Tool advisories are shown next to the flight strips (see Fig. 3).

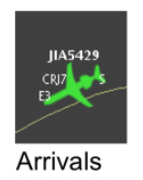

are green

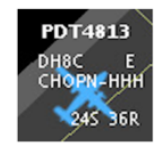

Westbound departures are brown, eastbound are blue

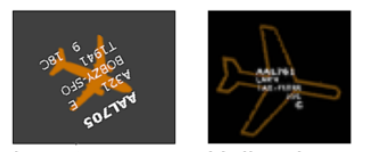

Hollow icon if no track

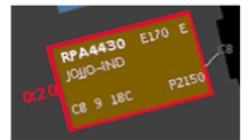

Flight on hold has red border

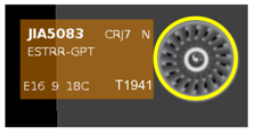

After pushback, engine symbol indicates spool up state

Fig. 2. Different states for flight strips and icons on RTC

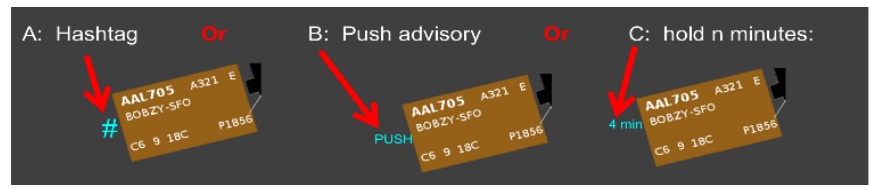

Fig. 3. Metering Tool advisories on RTC

\subsection{Ramp Manager Traffic Console (RMTC)}

The ramp manager has the ability to enable Time-Based Metering, i.e., the Metering Tool, via the user interface provided by the RMTC (see Fig. 4). The RMTC interface is used to set the Airport Movement Area (AMA) excess queue time using this interface. The target of 12 min excess queue time means that flights will be taking up to $12 \mathrm{~min}$ of predicted excess taxi time (or delay) in the airport movement area and any remaining time at the gates. The higher the excess queue time, the lower the amount of gate hold taken by that flight. These values for the target, upper and lower thresholds were investigated and determined in a HITL at NASA Ames [8]. These target excess queue values affect the output of the metering tool and its pushback advisories shown on the RTC.

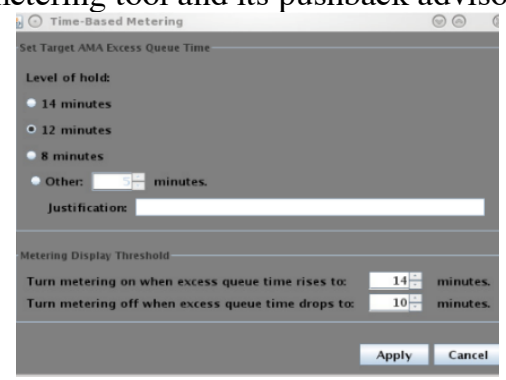

Fig. 4. Window on RMTC to set time-based metering and level of AMA Excess Queue Time 


\subsection{Tactical Scheduler for Surface}

The surface metering tool calculates Target Off-Block Times (TOBT) and provides gate hold recommendations to the ramp controller. For each departure flight, the tactical scheduler generates the Target Takeoff Time (TTOT) that would meet constraints, including runway separation criteria and Traffic Management Initiatives (TMI) constraints. Once the TTOT is generated, the TOBT is calculated according to the delay propagation formula: $T O B T=\max [E O B T, T T O T-U T T-$ MeteringValue $]$ where UTT is the unimpeded transit time from gate to runway. After calculating the TOBT, the TMAT is scheduled as TMAT $=$ TOBT + URT, where URT is the unimpeded ramp transit from gate to spot. This results in all the delay being absorbed in the AMA and no delay modeled or taken in the ramp.

Based on the flight's TOBT, a gate hold recommendation is provided on the RTC. The Metering Value or excess queue time is specified in the tactical scheduler's delay propagation logic and is used for calculation of TMATs and TOBTs. The purpose of the metering value is to control the maximum amount of predicted excess queue time that the flights are allowed to experience. The larger delay buffer causes the flights to spend more time in the queue or AMA before takeoff, and therefore, allows the aircraft to push back earlier from the gate. The target excess queue time (as shown in Fig. 3) selected by the ramp manager determines the value of delay buffer. The metering tool considers the demand and capacity imbalance for each departure runway before providing guidance for the flights. It is often observed that sometimes there are no gate hold advisories provided for flights departing in one direction, whereas gate hold advisories are provided for flights departing in the other direction.

Flights can be marked as exempt from metering or as a priority flights on the RTC or the RMTC, and the metering tool treats them accordingly. International and General Aviation (GA) flights may also be marked as exempt from metering. The tactical scheduler regards EOBT as a flight's predicted ready time and uses that to generate gate hold advisories. EOBT is being calculated by the airline based on various factors such as percentage of passengers boarded, baggage loaded and more.

The tactical surface scheduler allocates runway departure slots on the timeline according to the flight's schedule, with an order of consideration applied, and based on the quality of the flight's EOBT. The order of consideration dictates in the tactical surface scheduler the order in placing flights in different sequential groups based on their predictability in runway time prediction, which improves as the flight progresses in departure process, since the look-ahead time to the runway decreases. The definitions of the groups are shown in Table I.

Table 1. Definitions of Scheduling Groups

\begin{tabular}{|c|c|c|}
\hline Group & Definition & Interface (Fig. 4) \\
\hline Uncertain & $\begin{array}{l}\text { Flights with poor quality EOBT or EOBT - } \\
\text { current time }>10 \mathrm{~min}\end{array}$ & Hashtag \\
\hline Planning & $\begin{array}{l}\text { Flights within } 10 \mathrm{~min} \text { of EOBT (i.e., EOBT } \\
- \text { current time }<=10 \mathrm{~min} \text { ) }\end{array}$ & Advisory \\
\hline Ready & $\begin{array}{l}\text { Flights that have called in ready for } \\
\text { pushback }\end{array}$ & Advisory \\
\hline Out & Flights that are in pushback state & Engine symbol \\
\hline
\end{tabular}




\begin{tabular}{llc}
\hline Taxi & Flights that are cleared for taxi & Hollow or solid Aircraft icon \\
Queue & Flights waiting in the runway queue & Solid Aircraft icon \\
\hline
\end{tabular}

Flights that are further than 10 minutes from their EOBTs or have poor quality EOBTs (i.e., high prediction errors) are marked in the Uncertain group. The flight is considered to be part of the Planning group when it is 10 minutes from its EOBT. Gate hold advisories or push advisories will be shown on the RTC for the flights in the Planning group. When the pilot calls in ready to push, the ramp controller is expected to mark the flight strip for pushback or hold according to the advisory shown on the display, and at this point the scheduler marks the flight in the Ready group. When the flight is cleared for pushback by the ramp controller, it is considered to be in the Out group, and in the Taxi group when it starts taxiing. Similarly, it is considered in the Queue group when it is waiting in a queue at the runway getting ready for take-off. The RTC shows a hashtag for flights in the Uncertain group (Fig. 4) instead of providing any gate hold advisories. This is done to avoid too many fluctuations in the gate hold advisories due to the uncertainty in flight ready time. However, this does not prevent a pilot from calling in for pushback. When this happens, the ramp controller can click the hashtag, and the tactical scheduler instantaneously returns the gate hold advisory and displays it on the RTC. Flights automatically move from the Ready or Planning group to the uncertain group, if the flight called ready to push but did not pushback 5 minutes after its TOBT or it did not call 5 min past its EOBT respectively. The tactical surface metering tool frequently updates (i.e., every 10 seconds) and adjusts the schedule to accommodate uncertainties and changes in the traffic situation.

\subsection{Data Analysis and System Health (DASH)}

Data Analysis and System Health is a tool that provides several metrics in real time. This tool is used to look at predicted excess queue times in future, manipulate the target, upper, and lower thresholds for the excess queue times and see how many flights will be impacted when metering is enabled. Fig. 5 shows the ability to manipulate the thresholds in the bottom bar of the graph. The green middle line depicts the target excess queue time, the top red line shows the upper threshold for the excess queue time and the bottom line shows the lower threshold for excess queue time. The flights are depicted as dots on this chart and the number of dots above the upper threshold will likely have a gate hold time displayed as a pushback advisory, and those below the lower threshold do not display a pushback advisory on the RTC. When metering is enabled and advisories are being shown, every aircraft going to a runway that is being metered will show an advisory. For aircraft in this figure that are below the target queue, the advisory will be such that TOBT is the same as EOBT. For aircraft above the target queue, the advisory will be such that TOBT is greater than EOBT. 


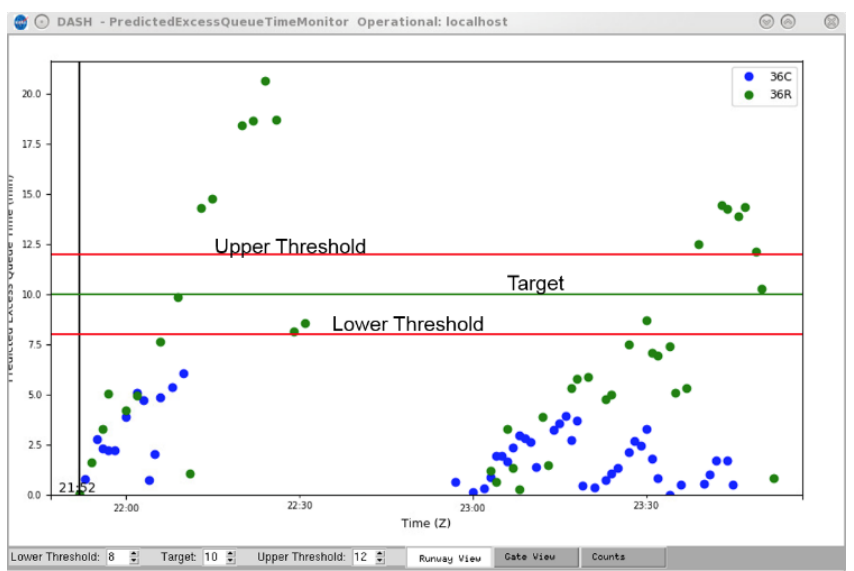

Fig. 5. Predicted Excess Queue Time Monitor (DASH) used for assessing impact of target values and thresholds.

\section{$4 \quad$ Metering Procedures at CLT}

\subsection{Initial Metering Procedures deployed at CLT}

In the Fall of 2017, Time-Based Surface Metering was introduced at CLT airport. The user interfaces via the RMTC and DASH were used collaboratively to enable metering by the user groups that comprised of the Ramp Manager and FAA Air Traffic Control (ATC) Tower's Traffic Management Coordinators (TMCs). The procedures developed with users at CLT started with asking the users to check in with each other before the start of the second departure push or Bank-2 of the day beginning around 0900 local time. In this verbal discussion session, the ramp mangers and TMCs discussed the demand and capacity imbalance situation by using the predicted excess queue time graphs in the DASH tool opened inside a "Metering What-if" system. They collaboratively determined the desired peak hold level and modified the target, upper and lower thresholds of excess queue time in the DASH tool. At about 0910, the Ramp manager verified the runway configuration and utilization plan intended for the upcoming Bank-2. They ensured that the airport and runway utilization scheme was an input into the ATD-2 tools so that tactical surface scheduler would have the correct predictions for capacity. The Ramp Manager enabled metering and was also responsible for making entries for the target, upper and lower thresholds in the RMTC. Once metering was enabled, all ATD-2 tools in the ramp and ATC Tower received a notification about the same. At this point, Ramp Managers also ensured that heavy jet aircraft and international flights were exempted from Metering.

\subsection{Initial Data Analysis and Feedback from Users at CLT}

This section describes the verbal feedback received from the users and some data analysis that depicts the changes made to the procedures early on. The data analyses were 
performed on data collected over a two-month period from November 29, 2017 to January 29,2018 . If metering was not used on particular days or if it was not collected correctly, those days were eliminated from these analyses. Although data was analyzed for both South and North Flows, only North Flow data is being reported here since it was the predominant configuration that the airport was in during the two-month period.

Initial Gate Hold Recommendations: Initially, the ramp controllers and managers indicated that when Surface Metering was enabled they observed that flights received gate hold recommendations even when there was little or no delay on the surface. The ramp controllers are used to pushing flights early rather than late to make room for arrivals, the early recommendations regarding gate hold provided guidance that was contrary to their usual way of operating. On further investigation, it was found that the system was only considering the predicted queue for all aircraft at the gate whose best Estimated Off Block Time was within 10 min of current time. Since the actual physical queue was not considered in this calculation, metering was triggered early based on the algorithm's predictions. This did not allow the queue to properly build up. Also the advisories correctly computed gate hold times for aircraft against EOBT but this alarmed the controllers since they did not expect to hold those flights during slow traffic.

To allow flights to pushback early in the bank and not be held when there was no traffic delay on the airport surface, the ramp managers were guided to make the upper threshold as +3 or +5 minutes above the target excess queue time (Fig. 6 shows the target and threshold values for the inputs that the users made). The high upper threshold values ensured that even though metering was enabled early on, display of pushback advisories was not triggered until the predicted excess queue time built up above the upper threshold, which meant that the onset of pushback advisories was delayed. Fig. 8 shows that metering was mostly triggered within 20 minutes from the time metering was enabled, which was usually earlier or close to the start of Bank-2, i.e., around 0900 local time. This confirmed the problem the ramp controllers reported regarding the gate hold recommendations coming early in the bank. A change in the metering algorithm was made and included in a subsequent software release. In addition to the predicted queue above the upper threshold for all aircraft at the gate whose UOBT was within 10 min of current time, the new metering algorithm also detected an active aircraft that was off the gate with a queue time greater than or equal to the target excess queue time. This change led to flights experiencing gate holds later in the bank as shown in Fig. 8. Figure 7 also shows that the values of the target, upper and lower thresholds input into the system after the algorithm change were lowered, since it was no longer necessary to have a high upper threshold with the change in the display triggering logic.

It was also reported by the users in the field that many flights depicted an advisory or a recommended gate hold time that was regarded as very high. This was the case because these flights had their ready times several minutes away, and the system had calculated their TOBT as greater than EOBT. For example, if an aircraft is being scheduled with an 8-minute gate hold (i.e., TOBT is 8 minutes after EOBT) and EOBT is 7 minutes in the future, this will result in an $8+7=15$ min advisory. In this case, ramp managers were guided to advise the ramp controllers to take a look at the ready time or EOBT in the flights strips on RTC via the Flight Menu. The Flight Menu is available one level deep inside the flight strip, and can be accessed by double clicking on the flight to open its flight menu. This information helped the ramp controllers to decide 
when to push the flights if the flights called earlier than their EOBT, given that airline policy does not expect flights to pushback earlier than 5 min to scheduled departure time. Several user interface changes were also explored to address this problem of not showing the ready times or EOBTs to the users in the flight strips but none have been implemented yet since the EOBT accuracy is variable.

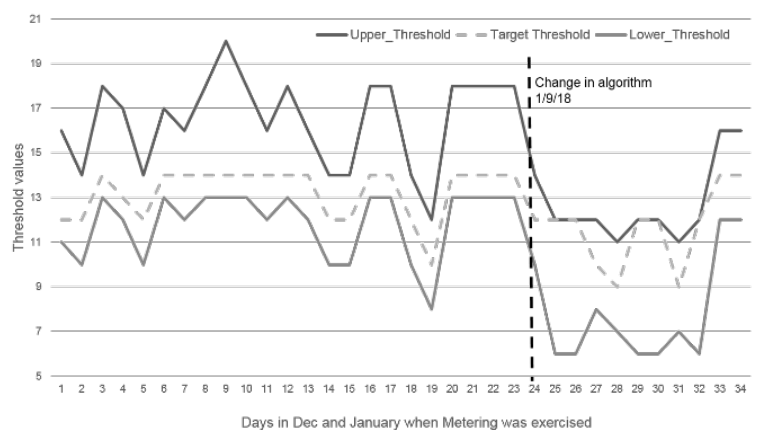

Fig. 6. Target and Thresholds set by users at CLT (north Flow only)

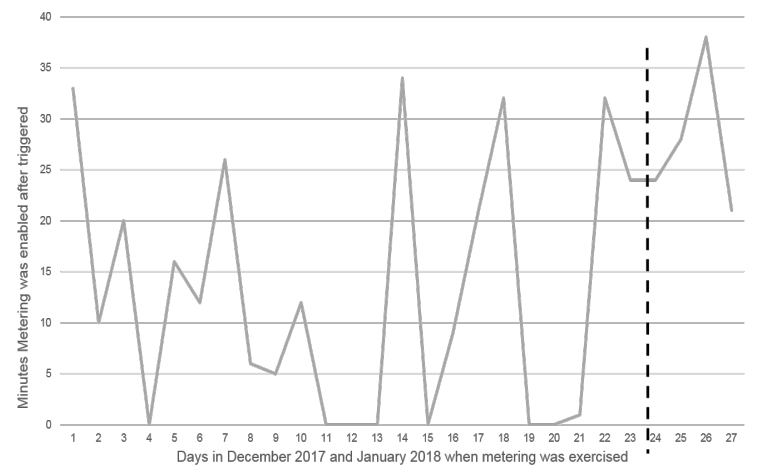

Fig. 7. Time gate-hold advisories were triggered after metering was enabled for runway $36 \mathrm{C}$ (north flow)

Number of flights impacted by Metering. The total number of flights impacted by metering is shown in Fig. 8. The number of flights subjected to metering is much higher than the flights that were actually held at the gates by the ramp controllers. The flights are considered as subject to metering when the pilot calls, metering is enabled and advisories are shown for the runway the aircraft was scheduled for. An advisory as mentioned earlier could be a push advisory or one with a recommended gate hold time. The number of flights actually held for metering is lower than the flights subjected to metering since the ramp controllers were allowed to push the flights up to 2 minutes before the recommended gate hold expired. Also in the early days of implementation the advisories were not followed due to the flights being held early in the bank when there was little or no delay in the surface traffic. 


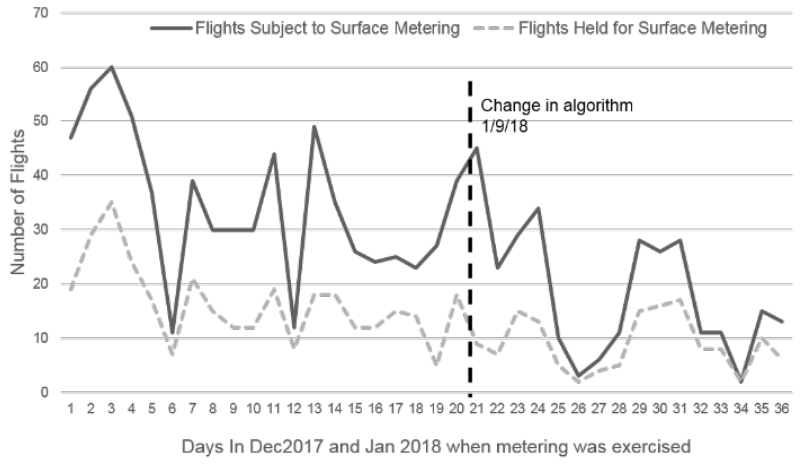

Fig. 8. Number of Flights impacted by metering for the entire airport

Gate Hold Advisories. The recommended gate hold or pushback advisories are shown in Fig. 9. The mean hold advisory which is calculated as time when the pilot calls in ready to pushback subtracted from the target off block time, is below 10 minutes for the most part with a few outliers. The ramp controllers actually held the flights less than 10 minutes. They were provided guidance to pushback flights within two minutes of the advisory. The peak advisories are a function of many different factors such as traffic demand, surface congestion, and whether or not aircraft called ready while in the uncertain group. If an aircraft that is in the uncertain group (Table1) calls the ramp during peak traffic, it is very likely they will receive a large hold advisory. The peak advisories have to be evaluated in light of the number of flights that were subject to metering.

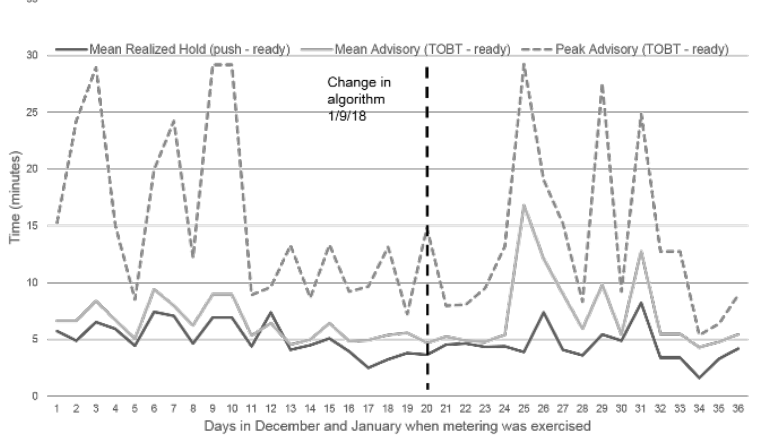

Fig. 9. Recommended Gate Hold Advisories shown during the two-month period

\section{Summary}

The Metering tool was deployed at CLT in the Fall of 2017 and metering procedures were collaboratively planned by the airline ramp control and FAA's ATCT. They collaboratively selected the target excess queue time that was used as input into the system. The initial deployment revealed an issue with the metering tool holding flights earlier 
in the bank when there was minimal delay associated with active flights on the surface, which was not operationally desired. The users were instructed to manage this situation by selecting higher upper threshold values. The algorithm was changed to ensure that not only predicted excess queue time but also actual queue time was taken into account for flights that were off the gate. This helped solved several problems, such as unnecessary gate hold times assigned to flights early in the bank. The users required refresher training to remind them to try to pay attention to the EOBTs and follow the advisories and airline policy regarding departures calling in early. This research brought a paradigm change for the controllers, who started paying attention to EOBTs and advisories instead of pushing every flight as they called thus reducing surface congestion. The algorithm change is expected to improve the compliance to the advisories, and hence reduce congestion. Future versions of this algorithm, which NASA continues to test in CLT, will focus on trying to meet target movement area entry times or spot times and not just pushback times.

\section{References}

1. S. Stroiney, B. Levy, H. Khadilkar, and H. Balakrishnan, "Assessing the impacts of the JFK ground management program," 32nd Digital Avionics Systems Conference (DASC), Syracuse, NY, October 2013.

2. Y. Jung, W. Malik, L. Tobias, G. Gupta, T. Hoang, et al., "Performance evaluation of SARDA: an individual aircraft-based advisory concept for surface management," Air Traffic Control Quarterly, Vol. 22, Number 3, 2015, pp. 195-221.

3. M. Hayashi, T. Hoang, Y. Jung, M. Malik, H. Lee, et al., "Evaluation of pushback decisionsupport tool concept for Charlotte Douglas International Airport ramp operations," 11th USA/Europe Air Traffic Management R\&D Seminar (ATM2015), Lisbon, Portugal, June 23-26, 2015.

4. Jung, Y., Engelland, S., Capps, A., Coppenbarger, R., Hooey, B., Sharma, S., Stevens, L., Verma, S., Lohr, G., Chevalley, E., Dulchinos, V., Malik, W., and Ruszkowski, L., "Airspace Technology Demonstration 2 (ATD-2) Phase 1 Concept of Use (ConUse)," NASA/TM-2017-xxxxxx, August 2017 (in review).

5. S. Engelland, A. Capps, K. Day, M. Kistler, F. Gaither, et al., "Precision Departure Release Capability (PDRC) final report," NASA/TM-2013-216533, June 2013.

6. J. Thipphavong, J. Jung, H. Swenson, K. Witzberger, L. Martin, et al., "Evaluation of the controller-managed spacing tools, flight-deck Interval management and terminal area metering capabilities for the ATM Technology Demonstration \#1," 10th USA/Europe ATM R\&D Seminar (ATM2013), Chicago, Illinois, 10-13 June 2013.

7. FAA Air Traffic Organization Surface Operations Directorate, "U.S. Airport Surface Collaborative Decision Making Concept of Operations (ConOps) in the Near-Term: Application of the Surface Concept at United States Airports," July, 2013.

8. Verma, S.; Lee, Hanbong, Martin, L., Stevens, L. Jung, Y., Dulchinos, V., Chevalley, E., Jobe, K., Parke, B. et al., "Evaluation of a Tactical Surface Metering Tool for Charlotte Douglas International Airport via Human-In-The-Loop Simulation," $36^{\text {th }}$ Digital Avionics Systems Conference (DASC), St. Petersburg, FL, Oct 2017

9. Charlotte Douglas International Airport, Fast Facts and Aviation Activity Reports, April 2017. http://www.cltairport.com/News/Pages/FactsandFigures.aspx 